\title{
Intragenic suppressors of a mutation in the aspartate chemoreceptor gene that abolishes binding of the receptor to methyltransferase
}

\author{
Daisuke Shiomi, Michio Homma and Ikuro Kawagishi
}

Division of Biological Science, Graduate School of Science, Nagoya University, Chikusa-ku, Nagoya 4648602, Japan
Author for correspondence: Ikuro Kawagishi. Tel: +81 52789 2993. Fax: +8152789 3001. e-mail: i45406a@nucc.cc.nagoya-u.ac.jp

In the chemotaxis of Escherichia coli, receptor methylation is the key process of adaptation. The methyltransferase CheR binds to the carboxy-terminal NWETF sequence of major chemoreceptors. The substitution of Ala for Trp of this sequence (W550A) of the aspartate chemoreceptor (Tar) abolishes its CheRbinding ability. In this study, six independent intragenic suppressors of the mutation were isolated. They were divided into two classes. Tar carrying the class I suppressors (G278A-L488M, T334A, G278A, G278C and A398T) showed signal biases toward tumbling, corresponding to increased activities of the receptor-associated histidine kinase CheA. These suppressors further reduced the unstimulated methylation level of Tar-W550A, but allowed slight but significant stimulation of methylation by aspartate. Some other CheAactivating mutations were also found to serve as class I suppressors. These results suggest that the class I suppressors compensate for the signal bias of Tar-W550A caused by its low methylation level and that the NWETF sequence is required primarily to maintain an appropriate level of methylation by increasing the local concentration of CheR around the receptor. The class II suppressor was a mutation in the termination codon (Op554W) resulting in the addition of 11 residues containing an XWxxF motif. This revertant Tar supported chemotaxis and was methylated almost as effectively as wild-type Tar. This effect was reversed by introducing a mutation in the $\mathrm{xWxxF}$ motif. These results reinforce the importance of the $\mathrm{XWXxF}$ motif and suggest that the motif does not have to be located at the extreme carboxy terminus.

Keywords: adaptation, chemotaxis, histidine kinase, methylation, revertant

\section{INTRODUCTION}

Cell-surface receptors receive extracellular signals (e.g. by binding ligands) and transduce them into intracellular signals (e.g. activation or inactivation of kinases). In many sensory systems, the receptors serve as targets of regulation (typically via covalent modification) for adaptation or desensitization, which is essential for highly sensitive detection of stimuli over a wide range.

Adaptation through covalent modification of receptors is essential for bacterial chemotaxis (for reviews, see Manson, 1992; Parkinson, 1993; Blair, 1995; Falke et al., 1997; Stock \& Surrette, 1996). Escherichia coli and Salmonella enterica serovar Typhimurium have several chemoreceptors that are reversibly methylated upon adaptation. These chemoreceptors are homodimeric proteins (Milligan \& Koshland, 1988), each subunit of which consists of two membrane-spanning regions, a periplasmic ligand-binding domain and a cytoplasmic signalling and adaptation domain. In the adaptation process, four to five glutamate residues (present in the motif Glu-Glu-X-X-Ala-Ser/Thr, with the underlined Glu residue being the site of methylation) in two different helices in the cytoplasmic domain are methylated or demethylated. These reactions are catalysed by the methyltransferase CheR and the methylesterase CheB, respectively. CheR catalyses transfer of a methyl group from $S$-adenosylmethionine to the side-chain carboxyl of glutamate residues (Springer \& Koshland, 1977).

The receptor forms a ternary complex with a cytoplasmic histidine kinase (CheA) and an adaptor protein (CheW) (Gegner et al., 1992; Schuster et al., 1993; Liu et al., 1997). The unliganded (or repellent-bound) receptor stimulates the activity of CheA, which auto- 
phosphorylates itself and then transfers the phosphate group to the response regulators $\mathrm{CheY}$ and CheB (Hess et al., 1988; Stock et al., 1988; Wylie et al., 1988). Phospho-CheY binds to FliM, which is a component of the flagellar motor, and induces the clockwise rotation of the motor, which causes tumbling (Barak \& Eisenbach, 1992; Welch et al., 1993). Dephosphorylation of phospho-CheY is facilitated by CheZ. Phospho-CheB catalyses demethylation of the receptor (Anand et al., 1998; Lupas \& Stock, 1989). When attractant binds to a chemoreceptor, it inhibits the activity of CheA (Borkovich et al., 1989). Consequently, the concentration of phospho-CheY decreases and the flagella rotate counterclockwise, which results in smooth swimming (Barak \& Eisenbach, 1992; Welch et al., 1993). The inhibition of the CheA kinase also decreases the concentration of phospho-CheB, the active form of the methylesterase, which reduces demethylation activity and hence increases methylation of the receptors. Methylation of a chemoreceptor stimulates CheA to counteract the inhibitory effect of the attractant (Borkovich et al., 1989).

The structure of the cytoplasmic domain of Salmonella Tar and its relation to kinase regulation were thoroughly studied by cysteine and disulfide scanning (Bass \& Falke, 1998, 1999; Bulter \& Falke, 1998; Danielson et al., 1997). Recently, the three-dimensional structure of a cytoplasmic fragment of $E$. coli $T$ sr that can activate the CheA kinase was determined (Kim et al., 1999). The cytoplasmic fragment (residues 286-526, corresponding to residues $284-528$ of Tar) has a highly $\alpha$-helical structure: each subunit consists of a coiled coil of two long, antiparallel helices (MH1 and $\mathrm{MH} 2$ ) connected by a U-turn, and the two subunits of a dimer form a quasifour-helix bundle. Methylation is predicted to alter the interaction between these cytoplasmic helices to switch signalling states.

A single cell contains thousands of chemoreceptors, but only hundreds of CheR molecules (Simms et al., 1987). How can the receptors be effectively methylated? The Cterminal pentapeptide sequence of chemoreceptors is well conserved among the high-abundance receptors (Tsr, Tar and Tcp), with the consensus being Asn-TrpGlu-Thr(Ser)-Phe. This motif is not conserved in the low-abundance receptors (Trg and Tap). CheR binds to this pentapeptide, which is distant from the methylation sites (Wu et al., 1996; Djorjevic \& Stock, 1998). The low-abundance receptors have weaker abilities to be methylated and to allow adaptation when they exist in a cell as sole chemoreceptors (Springer et al., 1977; Hazelbauer \& Engström, 1980; Yamamoto et al., 1990; Feng et al., 1997; Weerasuriya et al., 1998). However, when high-abundance and low-abundance receptors are co-expressed in a cell, the methylation level of the latter is enhanced (Kondoh et al., 1979; Yamamoto et al., 1990). Furthermore, the addition of the NWETF sequence to Trg enhances the methylation and adaptation abilities of that receptor (Feng et al., 1999). Finally, Barnakov et al. (1999, 2001) recently showed that CheB also binds to the NWETF motif. Taken together, the pentapeptide sequence seems to play a crucial role in receptor methylation and adaptation.

Recently, we systematically mutagenized this motif of Tar and showed that W550 and F553 (especially W550) play critical roles in the interaction with CheR, probably through hydrophobic interactions (Shiomi et al., 2000). Because CheR binding to Tar is almost completely eliminated by the W550A substitution, the methylation level of Tar-W550A is lower than that of wild-type Tar both in the absence and the presence of aspartate (Shiomi et al., 2000). However, methylation of Tar-W550A is still slightly enhanced by aspartate (Shiomi et al., 2000). The methylation level of Trg, which does not have a CheR-binding motif, is also regulated to some extent by its specific attractant ribose or galactose (Feng et al., 1999). To study the physiological role of the NWETF motif further, we isolated intragenic suppressors of the W550A mutation. Six suppressors were isolated and were divided into two classes: five class I suppressors, which generated a compensatory tumbling bias (CheA kinase-activating) and one class II suppressor that restored the affinity of Tar for CheR.

\section{METHODS}

Bacterial strains and plasmids. All E. coli K-12 strains used in the chemotaxis assay lack all four MCPs (i.e. Tsr, Tar, Trg and Tap) though they retain the related redox sensor Aer. Strain HCB339 $[\Delta($ tar-tap $) 5021 \Delta$ ts $r-7028$ trg:: Tn10 thr leu his met rpsL136] (Wolfe et al., 1987) has all of the Che proteins involved in general chemotaxis (i.e. CheA, CheW, CheY, CheZ, CheB and CheR). Strain HCB436 [ $\Delta t s r-7021 \Delta$ (tarcheB) $2234 \Delta t r g-100$ zbd:: Tn5 thr leu his met rpsL136] (Wolfe \& Berg, 1989) lacks CheB and CheR, and strain HCB1262 [ $\Delta$ (cheA-cheZ)::zeoR thr leu his met rpsL136] (Shiomi et al., 2000) lacks all the Che proteins.

Plasmids used in this work are listed in Table 1. The pBR322based plasmid pNI101 (Nishiyama et al., 1997) carries the wild-type tar gene. Plasmid pOKU121 (Shiomi et al., 2000) derives from pNI101 and encodes Tar-W550A. Plasmid pNT201 (Borkovich et al., 1989) carries the wild-type tar gene under the control of the tac promoter and was used to overexpress the Tar protein. Plasmid pKB23 (Borkovich et al., 1992) is based on pACYC184 and carries the wild-type cheR gene placed downstream of the tac promoter.

Construction of plasmids expressing mutant Tar proteins. Plasmids carrying class I suppressor tar genes without the original W550A mutation were constructed as follows. The $0.8 \mathrm{~kb}$ XbaI-MluI fragment from pDS303 (W550A-G278A) or pDS305 (W550A-G278C) was cloned into the corresponding region of pNI101 to yield pDS303W (G278A) and pDS305W (G278C), respectively. The $0.4 \mathrm{~kb}$ EcoT22I-PvuII fragment from pDS301 (W550A-G278A-L488M) was cloned into the corresponding region of pNI101 and pOKU121 to yield pDS311 (L488M) and pDS321 (W550A-L488M), respectively.

The $0.5 \mathrm{~kb}$ EcoR V-EcoT22I fragments containing the W192R, A198E, V201E, V202L, H267D or T303I mutations were cloned into the corresponding region of pNI101 or pOKU121 to yield pDS390W, pDS390A, pDS391W, pDS391A, pDS392W, pDS392A, pDS393W, pDS393A, pDS394W, pDS394A, pDS395W or pDS395A, respectively.

Plasmid pNI101 encoding wild-type Tar has two NdeI sites: 
Table 1. Tar-encoding plasmids used in this study

\begin{tabular}{|c|c|c|c|}
\hline Plasmid & Encoded Tar protein & Parent plasmid & Source \\
\hline pDS101 & Wild-type & pNT201 & This study \\
\hline pDS103 & W550A & pDS101 & This study \\
\hline pDS301 & G278A-L488M-W550A(original suppressor) & pOKU121 & This study \\
\hline pDS302 & T334A-W550A (original suppressor) & pOKU121 & This study \\
\hline pDS303 & G278A-W550A (original suppressor) & pOKU121 & This study \\
\hline pDS303W & G278A & pNI101 & This study \\
\hline pDS304 & W550A-Op554W (original suppressor) & pOKU121 & This study \\
\hline pDS3042 & W550A-Op554A & pOKU121 & This study \\
\hline pDS3043 & F553A-Op554W & pOKU151 & This study \\
\hline pDS305 & G278C-W550A (original suppressor) & pOKU121 & This study \\
\hline pDS305W & G278C & pNI101 & This study \\
\hline pDS306 & A398T-W550A (original suppressor) & pOKU121 & This study \\
\hline pDS311 & L488M & pNI101 & This study \\
\hline pDS321 & L488M-W550A & pOKU121 & This study \\
\hline pDS374 & W550A-Op554W & pDS101 & This study \\
\hline pDS390W & W192R & pNI101 & This study \\
\hline pDS390A & W192R-W550A & pOKU121 & This study \\
\hline pDS391W & A198E & pNI101 & This study \\
\hline pDS391A & A198E-W550A & pOKU121 & This study \\
\hline pDS392W & V201E & pNI101 & This study \\
\hline pDS392A & V201E-W550A & pOKU121 & This study \\
\hline pDS393W & V202L & pNI101 & This study \\
\hline pDS393A & V202L-W550A & pOKU121 & This study \\
\hline pDS394W & H267D & pNI101 & This study \\
\hline pDS394A & H267D-W550A & pOKU121 & This study \\
\hline pDS395W & T303I & pNI101 & This study \\
\hline pDS395A & T303I-W550A & pOKU121 & This study \\
\hline pDS396W & G278I & pNI101N & This study \\
\hline pDS396A & G278I-W550A & pOKU121 & This study \\
\hline pDS397W & G278R & $\mathrm{pNI} 101 \mathrm{~N}$ & This study \\
\hline pDS397A & G278R-W550A & pOKU121 & This study \\
\hline pDS398W & G278S & $\mathrm{pNI} 101 \mathrm{~N}$ & This study \\
\hline pDS398A & G278S-W550A & pOKU121 & This study \\
\hline pDS399W & G278T & pNI101N & This study \\
\hline pDS399A & G278T-W550A & pOKU121 & This study \\
\hline pNI101 & Wild-type & pAK101 & Nishiyama et al. (1997) \\
\hline $\mathrm{pNI} 101 \mathrm{~N}$ & Wild-type & pNI101 & This study \\
\hline pNT201 & Wild-type & pBR322 & Borkovich et al. (1989) \\
\hline pOKU121 & W550A & pNI101 & Shiomi et al. (2000) \\
\hline pOKU151 & F553A & pNI101 & Shiomi et al. (2000) \\
\hline
\end{tabular}

one is in the tar gene and the other is in the sequence derived from pBR322. The latter site was eliminated by blunt-ending and ligation to yield pNI101N. Site-directed mutagenesis of residue G278 was carried out by a two-step PCR method (Landt et al., 1990). The resulting $0.3 \mathrm{~kb}$ NdeI-EcoT22I fragments containing the G278I, G278R, G278S or G278T substitution were cloned between $\mathrm{NdeI}$ and EcoT22I sites of pNI101N to yield pDS396W, pDS397W, pDS398W or pDS399W, respectively. The $0.5 \mathrm{~kb} E c o R V-E c o T 22 \mathrm{I}$ fragments from pDS396W, pDS397W, pDS398W or pDS399W were cloned into the corresponding region of pOKU121 to yield pDS396A, pDS397A, pDS398A or pDS399A, respectively.

Plasmids overproducing Tar-W550A or W550A-Op554W were constructed as follows. The parental plasmid pNT201, which has two AvaI sites, was partially digested with AvaI, blunt-ended and ligated to yield pDS101. The $1.4 \mathrm{~kb} X b a \mathrm{I}-$ AvaI fragments from pOKU121 (W550A) or pDS304 (W550AOp554W) were cloned into the corresponding region of pDS101 to yield pDS103 (W550A) and pDS374 (W550AOp554W), respectively.

Swarm assay for chemotaxis. Swarm assays were performed with tryptone semisolid agar $(1 \%$ tryptone, $0.5 \% \mathrm{NaCl}, 0.3 \%$ agar) or minimal semisolid agar $[10 \mathrm{mM}$ potassium phosphate buffer $(\mathrm{pH} 7 \cdot 0), 1 \mathrm{mM}\left(\mathrm{NH}_{4}\right)_{2} \mathrm{SO}_{4}, 1 \mathrm{mM} \mathrm{MgSO}, 1 \mathrm{mM}$ glycerol, $1 \mu \mathrm{g}$ thiamine $\mathrm{ml}^{-1}, 0.1 \mathrm{mM}$ threonine, $0.1 \mathrm{mM}$ leucine, $0 \cdot 1 \mathrm{mM}$ histidine, $0 \cdot 1 \mathrm{mM}$ methionine, $0 \cdot 3 \%$ agar] supplemented with various concentrations of aspartate. When 
necessary, $50 \mu \mathrm{g}$ ampicillin $\mathrm{ml}^{-1}$ and/or $25 \mu \mathrm{g}$ chloramphenicol $\mathrm{ml}^{-1}$ was added. Cell suspensions $\left(2 \mu \mathrm{l}\right.$ each, about $4 \times 10^{6}$ cells) were spotted onto swarm plates, which were then incubated at $30^{\circ} \mathrm{C}$ for $10-20 \mathrm{~h}$.

Temporal assay of chemotactic response. Temporal stimulation assays for chemotaxis were carried out as described previously (Okumura et al., 1998). Cells were grown at $30^{\circ} \mathrm{C}$ in TG medium $[1 \%$ tryptone, $0.5 \% \mathrm{NaCl}, 0.5 \% \quad(\mathrm{w} / \mathrm{v})$ glycerol] supplemented, when necessary, with $50 \mu \mathrm{g}$ ampicillin $\mathrm{ml}^{-1}$ and/or $25 \mu \mathrm{g}$ chloramphenicol $\mathrm{ml}^{-1}$. Cells were harvested in late-exponential phase, washed with MLM medium $[10 \mathrm{mM}$ potassium phosphate buffer $(\mathrm{pH} 7 \cdot 0)$, $0 \cdot 1 \mathrm{mM}$ EDTA, $10 \mathrm{mM}$ DL-lactate, $0 \cdot 1 \mathrm{mM}$ methionine], resuspended in MLM medium and incubated at room temperature for $30 \mathrm{~min}$. For dose-response assays, the swimming patterns of the cells were observed with a dark-field microscope immediately after the addition of chemoeffector. For adaptation assays, a chemoeffector was added to the cell suspension, and small aliquots were taken at intervals for microscopic observation. The smooth-swimming fraction of the cells was measured using an Argus-10 image processor (Hamamatsu Photonics).

Analysis of receptor methylation by immunoblotting. Taking advantage of the fact that methylation of a receptor increases its mobility in SDS-PAGE (Boyd \& Simon, 1980; Chelsky \& Dahlquist, 1980; DeFranco \& Koshland, 1980; Engström and Hazelbauer, 1980), receptor methylation was examined with immunoblotting. HCB339 ( $\triangle \mathrm{MCPs}$ ) cells expressing the revertant Tar proteins were grown at $30{ }^{\circ} \mathrm{C}$ for $12 \mathrm{~h}$ in TG medium [ $1 \%$ tryptone, $0.5 \% \mathrm{NaCl}, 0.5 \%$ (w/v) glycerol] supplemented with ampicillin. The culture was diluted $1: 40$ into fresh TG medium supplemented with ampicillin. Cells were grown with vigorous shaking, harvested in the lateexponential phase and resuspended in $100 \mu \mathrm{l}$ distilled water. The samples were analysed by SDS-PAGE followed by immunoblotting with anti-Tsr (Iwama et al., 1997), which cross-reacts with Tar.

Analysis of receptor methylation by radiolabelling. Methylation of Tar was assayed by radiolabelling, as described previously (Nishiyama et al., 1997). Cells in medium consisting of $10 \mathrm{mM}$ potassium phosphate buffer ( $\mathrm{pH} 7 \cdot 0), 0 \cdot 1 \mathrm{mM}$ EDTA, $10 \mathrm{mM}$ DL-lactate, $3 \mu \mathrm{M}$ methionine and $0 \cdot 2 \mathrm{mg} \mathrm{ml}^{-1}$ chloramphenicol were incubated at $30^{\circ} \mathrm{C}$ with vigorous shaking. After $30 \mathrm{~min}, 3 \cdot 3 \mu \mathrm{M}\left[\right.$ methyl $\left.^{3} \mathrm{H}\right]$ methionine [8.5 $\mathrm{Ci} \mathrm{mmol}^{-1}\left(314.5 \mathrm{GBq} \mathrm{mmol}^{-1}\right)$; Amersham] was added to the cells and this suspension was shaken for another $30 \mathrm{~min}$. The cell suspension was divided into two aliquots, which were shaken for $15 \mathrm{~min}$ in the presence and absence of $10 \mathrm{mM}$ aspartate, respectively. The reaction was terminated by adding trichloroacetic acid, and methyl-labelled proteins were analysed by SDS-PAGE followed by fluorography.

\section{RESULTS}

\section{Isolation of intragenic suppressors of the W550A mutation}

When a plasmid encoding Tar-W550A is introduced into HCB339 $(\triangle \mathrm{MCP})$ cells, which lack all of the methylaccepting chemoreceptors except Aer, the resulting transformant cells respond to aspartate but do not adapt and hence cannot swarm in tryptone semisolid agar (Shiomi et al., 2000). Starting with this strain, we isolated 60 independent spontaneous pseudorevertants that

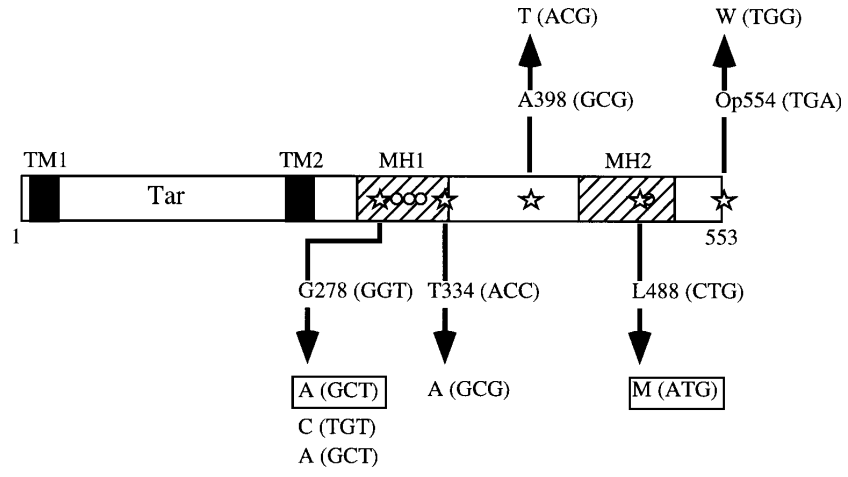

Fig. 1. Intragenic suppressors of the W550A mutation of tar. Among 60 independent swarm-positive revertants from HCB339 ( $\triangle M C P)$ cells carrying plasmid pOKU121 (encoding Tar-W550A), six proved to have mutations in the tar gene. They retained the original mutation and had the following second-site suppressor mutations: G278A, G278A-L488M, G278C, T334A, A398T and Op554W. The mutation sites are indicated with stars in the tar coding region with the original (with residue numbers) and the mutant amino acid residues (the corresponding nucleotide sequences are shown in parentheses). The double mutations (G278A-L488M) are marked with open boxes. Op554 indicates the termination codon (TGA), the mutation of which results in the addition of the 11 residues (WRVFTNAVIAA) to the $C$ terminus of Tar-W550A. Open circles indicate methylation sites. TM1 and TM2, the first and the second transmembrane helices; $\mathrm{MH} 1$ and $\mathrm{MH} 2$, the first and the second methylation helices.

spread in tryptone semisolid agar. Plasmid DNA prepared from these revertants was reintroduced into the host strain, and plasmids from six independent pseudorevertants gave swarm-positive transformants. The nucleotide sequences of the tar genes from the six revertants were determined. All of them retained the original W550A mutation and had second-site suppressors (Fig. 1): four of them were single missense mutations (G278A, G278C, T334A and A398T), one had two mutations causing two amino acid changes (G278A-L448M), and one had a mutation in the termination codon (Op554W: TGA to TGG) that resulted in the addition of 11 residues at the $\mathrm{C}$ terminus of Tar.

\section{Swarming abilities of cells expressing the revertant Tar receptors}

HCB339 cells expressing these revertant Tar receptors were tested for their swarming abilities in semisolid agar plates (Fig. 2). In tryptone semisolid agar, cells expressing the $\mathrm{Op} 554 \mathrm{~W}$ receptor formed a slightly larger swarm than cells expressing wild-type Tar. The cells expressing the other revertant Tar proteins formed smaller swarms than cells expressing wild-type Tar [Fig. $2 \mathrm{~A}, \mathrm{~B}(\mathrm{a})$ ]. Thus, the suppressors can be divided into two classes: five class I suppressors (G278A-L488M, T334A, G278A, G278C and A398T), which restore swarming only partially, and one class II suppressor (Op554W), which restores full swarming ability. Essentially similar 
A

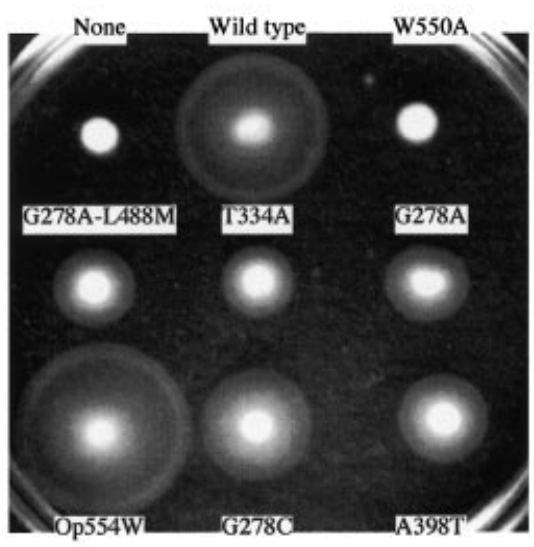

B

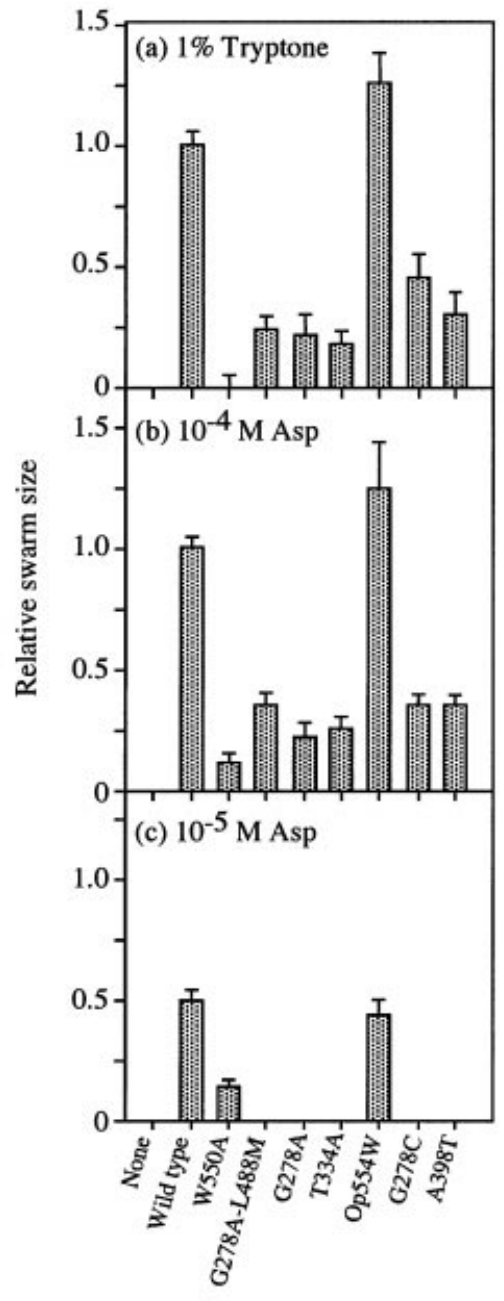

Fig. 2. Swarming ability of HCB339 cells expressing the revertant Tar receptors. Aliquots ( $2 \mu \mathrm{l}$ each) from three independent fresh overnight cultures were spotted onto ampicillin-supplemented tryptone [A, B(a)] or minimal semisolid agar containing $10^{-4} \mathrm{M}$ $[\mathrm{B}(\mathrm{b})]$ or $10^{-5} \mathrm{M}[\mathrm{B}(\mathrm{c})]$ aspartate and the plate was then incubated at $30^{\circ} \mathrm{C}$. The swarm diameter of cells carrying the vector pBR322 (none) was subtracted from those of cells expressing each receptor to obtain net swarm diameters. The relative swarm diameter is defined as the net swarm diameter normalized to the mean diameter of swarms formed by cells expressing wildtype $\operatorname{Tar}(\mathrm{B})$. The relative swarm diameters in the presence of $10^{-5} \mathrm{M}$ aspartate $[B(C)]$ were normalized to the mean diameter of swarms formed by cells expressing wild-type Tar in the presence of $10^{-4} \mathrm{M}$ aspartate $[\mathrm{B}(\mathrm{b})]$. results were obtained when minimal semisolid agar containing $10^{-4} \mathrm{M}$ aspartate was used [Fig. $\left.2 \mathrm{~B}(\mathrm{~b})\right]$. In contrast, in minimal semisolid agar containing $10^{-5} \mathrm{M}$ aspartate, cells expressing Tar-W550A formed a larger swarm than cells expressing any class I revertant Tar, though the swarm was much smaller than that of cells expressing wild-type Tar [Fig. 2B(c)]. It is possible that the gradient formed in agar containing $10^{-5} \mathrm{M}$ aspartate are not steep enough to generate smooth swimming with the class I revertants that cause tumbling bias (see below). On the other hand, the gradient formed with $10^{-4} \mathrm{M}$ aspartate may be steep enough to stimulate the class I revertants but may not allow cells expressing Tar-W550A to adapt.

\section{Adaptation abilities of cells expressing the revertant Tar receptors}

Swarming is influenced not only by the ability of cells to respond to and to adapt to attractants but also by cell growth and signal (smooth-swimming versus tumbling) bias. We therefore examined swimming bias and chemo- tactic responses of HCB339 cells expressing each revertant Tar in the temporal stimulation assay. The basal swimming bias of cells expressing Tar-W550A was similar to that of cells expressing wild-type Tar (Fig. 3A). However, they did not adapt to aspartate (Fig. 3A) even after $13 \mathrm{~min}$ (data not shown). We determined minimal aspartate concentrations that elicit $100 \%$ attractant responses mediated by the revertant proteins (data not shown). These concentrations were used in the following adaptation assays. Cells expressing any class I revertant Tar were tumbly (Fig. 3B). However, they became smooth-swimming immediately after addition of $10^{-5}$ or $10^{-4} \mathrm{M}$ aspartate (i.e. attractant responses) and they gradually adapted to return their original tumbly behaviours (Fig. 3B). In contrast, cells expressing the class II revertant Tar (W550A-Op554W) behaved similarly to cells expressing wild-type Tar both in terms of their basal swimming bias and adaptation (Fig. 3B). These results suggest that the class I suppressors bias the signal output toward tumbling (i.e. CheA-activating mutations) whereas the class II suppressor restore the affinity of Tar for CheR. 
A

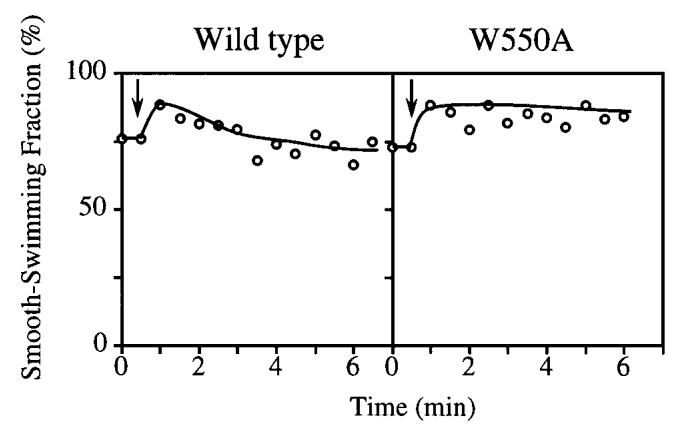

B

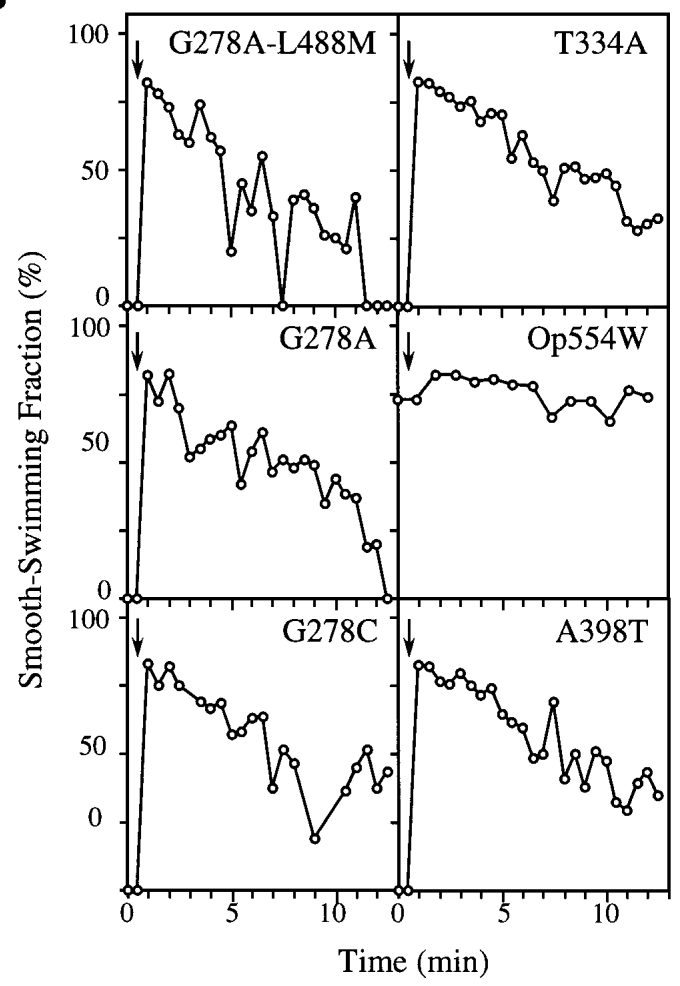

Fig. 3. Adaptation ability of $\mathrm{HCB} 339$ cells expressing TarW550A or its revertant derivatives. (A) Time-courses of aspartate responses of $\mathrm{HCB} 339$ cells expressing the wild-type or W550A receptor. At the time indicated by an arrow, $10^{-5} \mathrm{M}$ aspartate was added. (B) Time-courses of aspartate responses of HCB339 cells expressing each revertant receptor. Aspartate was added at $10^{-5} \mathrm{M}$ (G278A-L488M, G278A and Op554W) or $10^{-4} \mathrm{M}$ (T334A, G278C and A398T).

\section{Methylation patterns of the revertant Tar receptors}

Methylation of chemoreceptors is the key process of adaptation. HCB339 ( $\triangle \mathrm{MCP})$ cells expressing any of the revertant Tar proteins adapted to aspartate (Fig. 3B), whereas HCB436 cells, which lacks $\mathrm{CheB}$ and CheR as well as all four chemoreceptors $(\triangle \mathrm{MCP} \Delta \mathrm{CheB} \Delta \mathrm{CheR})$, did not adapt to aspartate when they expressed any of the revertant receptor proteins (data not shown), implying that methylation of all the revertant Tar proteins is modulated in response to aspartate. We therefore examined the methylation patterns of the revertant Tar proteins (Fig. 4). Although the expression level of the class II revertant Tar receptor was lower than that of wild-type Tar (Fig. 4A), the unstimulated (-) and aspartate-stimulated $(+)$ methylation levels of TarW550A-Op554W were similar to those of wild-type Tar (Fig. 4), supporting the notion that the class II revertant restores the affinity for CheR. In contrast, the methylation levels of the class I revertant Tar receptors were even lower than that of the original mutant Tar-W550A under both the unstimulated $(-)$ and the aspartatestimulated $(+)$ conditions (Fig. 4B). This result suggests that the class I revertants do not restore affinity for CheR. However, these revertants still showed slightly but significantly enhanced methylation in the presence of aspartate, a property consistent with their ability to adapt.

\section{Analysis of the class I suppressors}

To characterize the class I suppressor mutations further, we constructed Tar receptors carrying G278A or G278C without the original W550A substitution. HCB339 cells expressing Tar-G278A or G278C were then tested for chemotactic ability (Fig. 5A). These cells formed larger swarms than cells expressing the W550A receptor. Cells expressing Tar-G278A swarmed slightly faster than cells expressing Tar-G278C and as fast as cells expressing wild-type Tar. These cells responded and adapted to aspartate as well as cells expressing the corresponding receptor with W550A (data not shown). Immunoblotting with anti-receptor serum showed that the methylation patterns of the G278A and G278C receptors were similar to those of the corresponding receptors with W550A (data not shown). These results suggest that G278A and G278C are tumbling-bias mutations as Trammell \& Falke (1999) showed for Salmonella Tar. It is likely that T334A and A398T also cause a tumbling bias (see below).

Next, to examine the nature of the L488M mutation, we constructed plasmids encoding Tar carrying the L488M mutation with or without the W550A mutation. HCB339 cells expressing Tar-L488M swarmed as fast as cells expressing wild-type Tar, whereas cells expressing the corresponding receptor with W550A failed to swarm (Fig. 5A). This result indicates that the L488M mutation does not suppress the W550A mutation and was just a silent second change in the G278A-L488M receptor.

\section{Effects of kinase-activating mutations on the W550A receptor}

The results described above raised the possibility that other tumbling-bias (kinase-activating) mutations may also serve as class I suppressors of the W550A substitution. We therefore introduced several known tumbling-bias mutations into the W550A receptor. First, we focused on residue G278 in the first methylation helix because this residue is a critical site of kinase regulation 
A

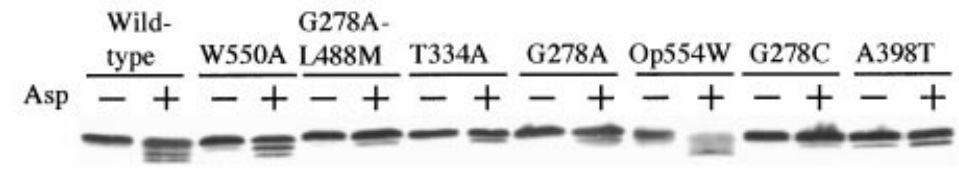

B

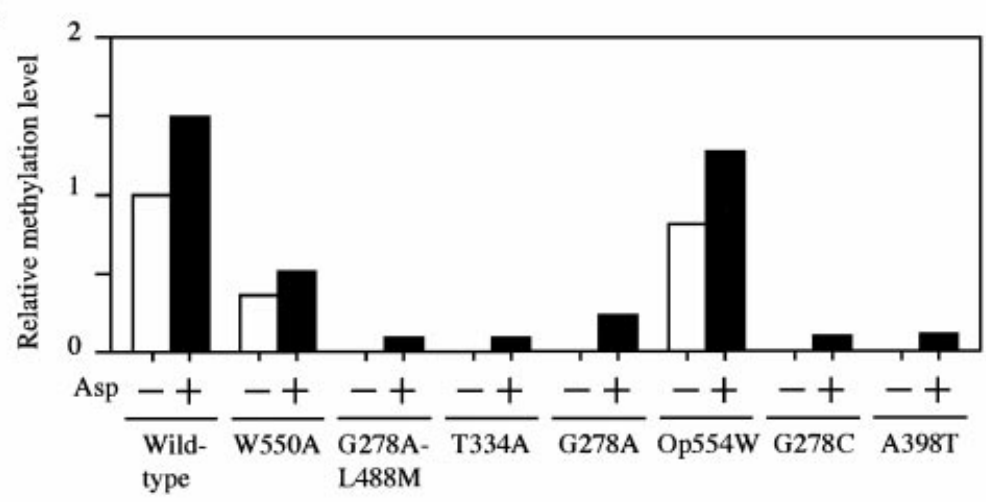

Fig. 4. Methylation levels of the revertant Tar receptors. Methylation of the wild-type, W550A, and revertant receptors was assayed by immunoblotting with anti-Tsr (A) and radiolabelling (B), as described in Methods, in the absence $(-)$ or presence $(+)$ of 10 $\mathrm{mM}$ aspartate. For each sample, the intensity of the receptor band on fluorographs was divided by that of methylatable EF-Tu protein, which serves as an internal control. The resulting value was normalized to that of wild-type Tar in the absence of aspartate to obtain the relative level of methylation.
(Trammell \& Falke, 1999). The residue was replaced by Ser, Thr, Arg or Ile. Cells expressing Tar-G278T-W550A or Tar-G278R-W550A formed a similar swarm to cells expressing the class I revertant Tar receptors, whereas cells expressing Tar-G278S-W550A formed a smaller swarm (Fig. 5B). Cells expressing Tar-G278I-W550A failed to swarm (Fig. 5B), which is consistent with the fact that the Tar-G278I mutant protein cannot regulate kinase activity in response to aspartate (i.e. it constitutively activates CheA kinase) (Trammell \& Falke, 1999).

Next, we examined the mutations that were initially isolated as suppressors of the A19K substitution that abolishes the signalling ability of Tar without impairing the aspartate-binding ability (Oosawa \& Simon, 1986). All of them are single-residue substitutions in the second transmembrane helix (TM2; W192R, A198E, V201E and V202L) or in the first methylation helix (MH1; H267D and T303I) that cause a wide range of tumbling biases (Tatsuno et al., 1996; Umemura et al., 1998). In the absence of chemoeffector, cells expressing TarA198E-W550A or Tar-T303I-W550A tumbled incessantly, whereas cells expressing the other mutant receptors showed various degrees of signal bias (Fig. 5C). In swarm assay, cells expressing Tar-A198E-W550A or Tar-T303I-W550A formed a similar swarm to cells expressing the class I revertant receptor, whereas cells expressing any of the other constructs failed to swarm (Fig. 5D). Thus, the A198E (in TM2) and T303I (in MH1) substitutions, but not the other substitutions which caused weaker tumbling biases, can serve as suppressors of the W550A substitutions. Taken together, a strong (but not constitutive) tumbling bias seems to be required (and probably sufficient) for suppression of the W550A substitution.

\section{Analysis of the class II suppressor}

The results described above suggest that the class II suppressor restores the affinity of Tar-W550A for CheR.
We tried to detect binding of CheR to inverted membrane vesicles containing Tar-W550A-Op554W. However, the level of binding was much lower than that to membranes containing wild-type Tar (data not shown). The class II revertant Tar receptor has 11 additional residues (WRVFTNAVIAA) due to the mutation of the termination codon $(\mathrm{Op} 554 \mathrm{~W})$. This carboxy-terminal extension contains an $\mathrm{xW} \mathrm{xxF}$ motif that is especially important for the interaction with CheR (Djordjevic \& Stock, 1998; Shiomi et al., 2000) and therefore might serve as a (weak) binding site for CheR. To examine this possibility, we first examined the effect of the Op554W mutation on the Tar receptor with the F553A mutation, which is less severe than W550A (Shiomi et al., 2000), probably because the phenylalanine residue is less critically involved in the interaction with CheR than the tryptophan residue (Djordjevic \& Stock, 1998). Cells expressing Tar-F553A-Op554W swarmed as fast as cells expressing Tar-W550A-Op554W (Fig. 6), indicating that the Op554W mutation can also suppress the defect caused by the F553A mutation. We next examined the effect of Op554A substitution (i.e. the loss of the $\mathrm{xW} \times \mathrm{xxF}$ motif in the 11 additional sequences) on the W550A receptor. Cells expressing Tar-W550A-Op554A failed to swarm, suggesting that the tryptophan residue at position 554 is critical for suppression. These results are consistent with the interpretation that the class II suppressor partially restores binding of CheR to the receptor by creating an $\mathrm{xW} \times \mathrm{xF}$ motif in the carboxyterminal additional sequence.

\section{DISCUSSION}

In this study, we isolated six independent intragenic suppressors from the mutant tar gene encoding the W550A residue substitution. These suppressors could be divided into two classes. When expressed as the sole chemoreceptors in a cell, the five class I revertant receptors supported chemotaxis less effectively than wild-type Tar. Their methylation levels were even lower 

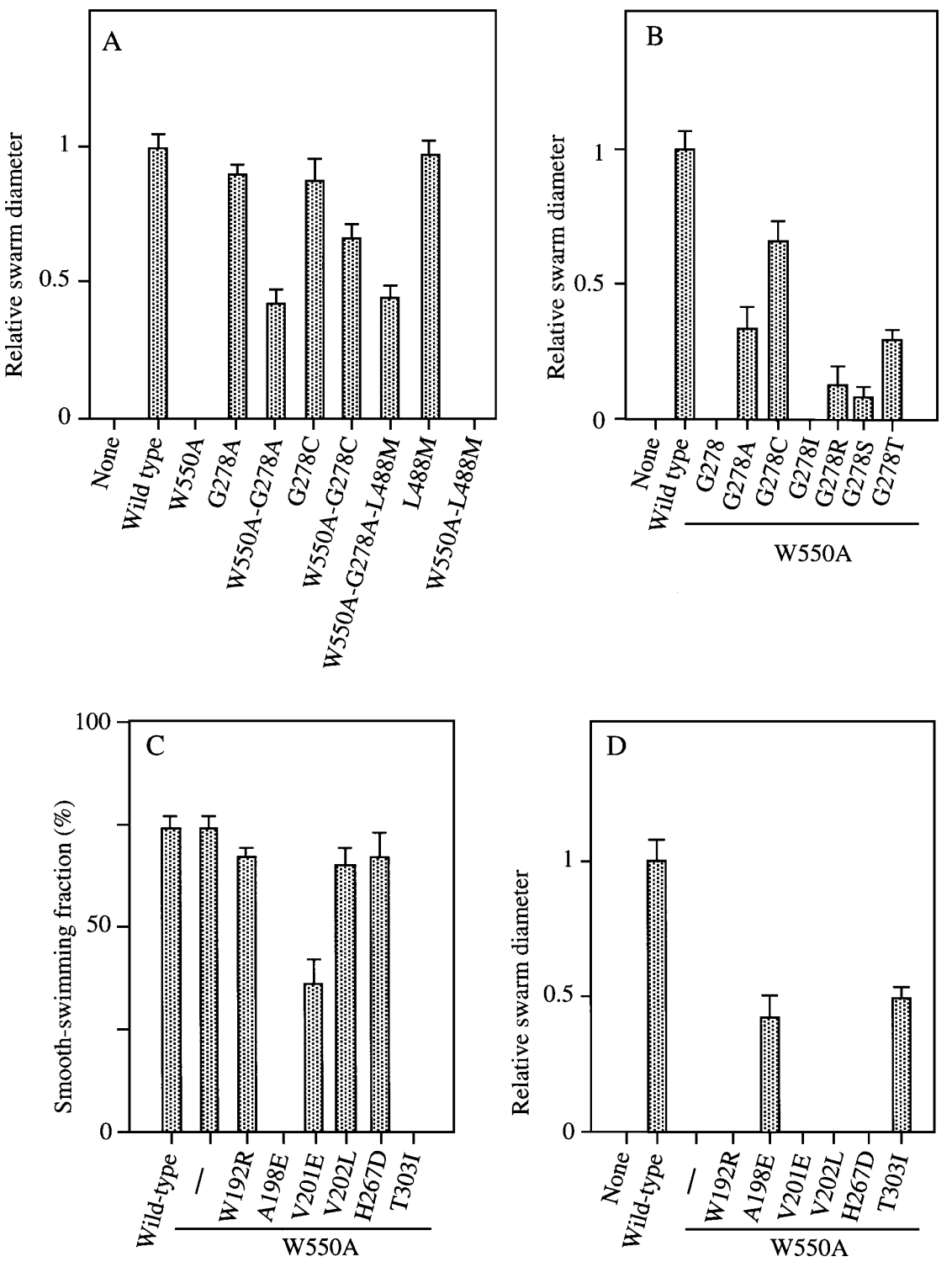

Fig. 5. Characterization of class I suppressors. (A) Swarming ability of HCB339 cells expressing the wild-type, W550AG278A-L488M，W550A-G278A， W550AG278C, G278A, G278C, L488M or no receptor (None). (B) Swarming ability of HCB339 cells expressing the $\mathrm{W} 550 \mathrm{~A}$ receptor carrying an additional tumbling-bias mutation (G278A, G278C, G278I, G278R, G278S, or G278T) as well as those expressing the wild-type, or no receptor (None). (C) Swimming bias of HCB339 cells expressing the W550A receptor with or without $(-)$ an additional A19Ksuppressor mutation (A198E, V201E, V202L, H267D, or T303I) as well as those expressing the wild-type receptor. (D) Swarming ability of HCB339 cells expressing the W550A receptor with or without an additional A19K-suppressor mutation (A198E, V201E, V202L, H267D, or T303I) as well as those expressing the wild-type, or no receptor (None). Swarming was tested in tryptone semisolid agar containing ampicillin. The relative swarm size is defined as in the legend to Fig. 2.

than that of Tar-W550A, but methylation levels increased significantly upon the addition of aspartate. The class II revertant receptor, in which the original termination codon was altered to a UGG (Trp) codon led to the addition of 11 additional residues, supported chemotaxis, and was methylated almost as effectively as wild-type Tar.

Of the class I suppressors, G278A and G278C had already been characterized by Trammell \& Falke (1999): the receptors with these mutations superactivate CheA autophosphorylation in the absence of aspartate, but in the presence of aspartate the mutant receptors fully and partially inhibit the kinase, respectively. The receptors with the other class I suppressors (T334A and A398T) also exhibited tumbling-biased signalling and their methylation levels were lower than that of Tar-W550A, suggesting that these mutations also render the signal bias toward the activation of CheA. We also showed that some other tumbling-bias mutations, which allow regulation of the activity of CheA kinase in response to aspartate, are also able to suppress the W550A suppression regardless of the place of the second site mutation. However, among the tumbling-bias mutations tested, those which cause weaker tumbling biases or which cause constitutive activation of CheA failed to suppress the W550A mutation. Therefore, we conclude that the class I suppressors compensate for an excessively smooth-swimming (i.e. CheA-inactivating) bias caused by decreased methylation of Tar-W550A.

Wild-type Tar inhibits CheA activity in the presence of aspartate, but increased methylation of Tar during adaptation to aspartate restores the CheA-stimulating activity. Methylation of Tar-W550A can also be stimulated to a certain extent by aspartate, but this enhancement of methylation may be too slight to reactivate CheA in the presence of aspartate. The class I revertant Tar receptors can inhibit the CheA activity in response to aspartate, but they still can stimulate CheA when their methylation is enhanced by aspartate (i.e. when the cell adapts to aspartate) although their absolute levels of 


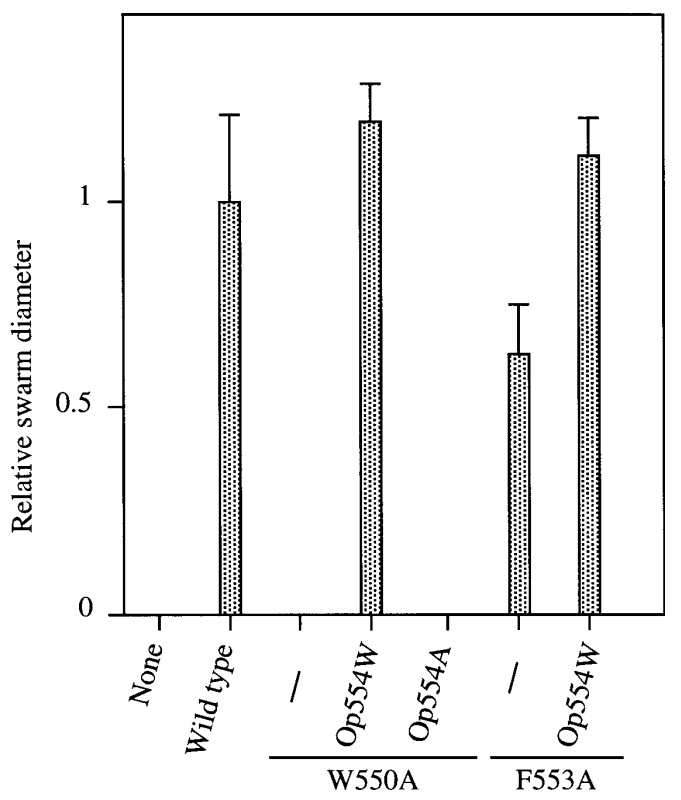

Fig. 6. Swarming ability of HCB339 cells expressing the W550A or F553 receptor with or without $(-)$ an additional mutation Op554W or Op554A as well as those expressing the wild-type, or no receptor (None). Swarming was tested in tryptone semisolid agar containing ampicillin. The relative swarm size is defined as in the legend to Fig. 2.

methylation are lower than that of wild-type Tar, presumably because of 'constitutive' activation of CheA, and hence the methylesterase CheB. This difference in CheA activation between the methylated and unmethylated ligand-occupied revertant receptors may be enough to support chemotaxis to some extent.

The mechanism by which the class I mutations suppress the defect introduced by the W550A substitution demonstrates the robustness of the chemotaxis system (Barkai \& Leibler, 1997; Alon et al., 1999). Alon et al. (1999) showed that chemotaxis is largely independent of the basal swimming bias and the adaptation time. Although the revertants failed to support swarming in minimal semisolid agar containing $10^{-5} \mathrm{M}$ aspartate, the requirement for higher concentrations of aspartate is readily explained by the suppression mechanism described above. The tumbling-biased signals produced by the class I receptors may be too strong to be suppressed by $10^{-5} \mathrm{M}$ aspartate.

Presumably, the class II revertant receptor partially restores binding of CheR to the receptor. When expressed as a sole chemoreceptor in a cell, this receptor supports chemotaxis and a wild-type signal bias, and it is methylated almost as effectively as wild-type Tar. We examined binding of CheR to inverted membrane vesicles containing the class II receptor, but the level of binding was much lower than to membranes containing wild-type Tar (data not shown), suggesting that the affinity of the revertant protein for CheR is weaker than that of wild-type Tar. This revertant Tar receptor has 11 foreign residues (WRVFTNAVIAA) due to the mutation of the termination codon (Op554W). The results presented here suggest that the class II suppressor partially restores binding of CheR to the receptor by creating an $\mathrm{xW} x \mathrm{xF}$ motif in the carboxy-terminal additional sequence. This means that the CheR-binding sequence may not have to be located at the extreme carboxy terminus of the chemoreceptor.

The current understanding of the regulation of methylation of chemoreceptors is as follows. Binding of an attractant to a chemoreceptor leads to inhibition of CheA (Borkovich et al., 1989), and hence decreases phosphorylation of CheB (Hess et al., 1988; Lupas \& Stock, 1989) to decrease the rate of demethylation. The attractant-bound receptor becomes a better substrate for CheR and a poorer substrate for $\mathrm{CheB}$, resulting in a net increase in methylation of the liganded receptor (Springer et al., 1977; Wang \& Koshland, 1980). Since the methylation levels of the class I revertant Tar proteins were lower than the methylation level of TarW550A, we conclude that the affinity of Tar for CheR was not restored by the class I suppressors. However, the methylation levels of these receptors were slightly but significantly enhanced by aspartate, suggesting that binding of CheR to the NWETF sequence is not strictly required for the regulation of the methylation level of the chemoreceptor.

What is the role of the pentapeptide sequence then? It is likely that the pentapeptide motif is required for maintaining an appropriate level of receptor methylation by increasing the local concentration of CheR around the receptor cluster in the cell. This interpretation is consistent with the following facts: (i) CheR is much less abundant than the receptor molecules (Simms et al., 1987); (ii) the overproduction of CheR suppresses the defect of the mutations in the pentapeptide sequence (Okumura et al., 1998; Shiomi et al., 2000); (iii) the receptor molecules form a cluster with $\mathrm{CheW}$ and CheA at a cell pole (Maddock \& Shapiro, 1993; Lybarger \& Maddock, 2000); (iv) a receptor dimer with the CheRbinding sequence can help methylation of a neighbouring receptor dimer without the CheR-binding sequence (Le Moual \& Koshland, 1997; Li et al., 1997); (v) GFP-CheR localized to cell poles in the presence, but not in the absence, of a chemoreceptor with the NWETF sequence (Shiomi et al., 2002).

Okumura et al. (1998) showed that cells expressing mutant Tcp (NWESLA) respond to, but do not adapt to the repellent glycerol. Barnakov et al. (1999, 2001) demonstrated that CheB also binds to the NWETF peptide. We also found that the W550A receptor serves as a poorer substrate of $\mathrm{CheB}$ (unpublished results). These findings suggest that the NWETF sequence serves also as a modulator of CheB activity. The intragenic suppressors of the W550A mutation isolated in this study are either CheA-activating mutations or a mutation that seemingly partially restores the affinity of Tar for CheR. Moreover, the same original mutation can be suppressed by the overproduction of CheR 
(Shiomi et al., 2000). Therefore, at least under the conditions tested, binding of the receptor to CheR may be more important (or rate-limiting) to support chemotaxis than that to CheB. It is also possible that the W550A mutation has a more severe effect on binding of CheR than that of CheB. Further analyses will be required to address these issues.

\section{ACKNOWLEDGEMENTS}

We thank Hisashi Okumura for examining conditions of revertant screening, Dr Tohru Umemura, Kenji Fukami and Tatsuya Furihata for helping with plasmid construction and nucleotide sequencing, and Dr Ken Sato for helpful discussion and technical advice. We thank Dr Michael D. Manson (Texas A \& M University) for critically reading the manuscript. This work was supported in part by grants-in-aid for scientific research from the Society for the Promotion of Science (to D.S. and I.K) and from the Takeda Science Foundation (to I.K.).

\section{REFERENCES}

Alon, U., Surette, M. G., Barkai, N. \& Leibler, S. (1999). Robustness in bacterial chemotaxis. Nature 397, 168-171.

Anand, G. S., Goudreau, P. N. \& Stock, A. M. (1998). Activation of methyltransferase $\mathrm{CheB}$ : evidence of a dual role for the regulatory domain. Biochemistry 37, 14038-14047.

Barak, R. \& Eisenbach, M. (1992). Correlation between phosphorylation of the chemotaxis protein $\mathrm{CheY}$ and its activity at the flagellar motor. Biochemistry 31, 1821-1826.

Barkai, N. \& Leibler, S. (1997). Robustness in simple biochemical networks. Nature 387, 913-917.

Barnakov, A. N., Barnakova, L. A. \& Hazelbauer, G. L. (1999). Efficient adaptational demethylation of chemoreceptors requires the same enzyme-docking site as efficient methylation. Proc Natl Acad Sci U S A 96, 10667-10672.

Barnakov, A. N., Barnakova, L. A. \& Hazelbauer, G. L. (2001). Location of the receptor-interaction site on $\mathrm{CheB}$, the methylesterase response regulator of bacterial chemotaxis. J Biol Chem 276, 32984-32989.

Bass, R. B. \& Falke, J. J. (1998). Detection of a conserved $\alpha$-helix in the kinase-docking region of the aspartate receptor by cysteine and disulfide scanning. J Biol Chem 273, 25006-25014.

Bass, R. B. \& Falke, J. J. (1999). The aspartate receptor cytoplasmic domain: in situ chemical analysis of structure, mechanism and dynamics. Structure 7, 829-840.

Blair, D. F. (1995). How bacteria sense and swim. Annu Rev Microbiol 49, 489-522.

Borkovich, K. A., Kaplan, N., Hess, J. F. \& Simon, M. I. (1989). Transmembrane signal transduction in bacterial chemotaxis involves ligand-dependent activation of phosphate group transfer. Proc Natl Acad Sci US A 86, 1208-1212.

Borkovich, K. A., Alex, L. A. \& Simon, M. I. (1992). Attenuation of sensory receptor signaling by covalent modification. Proc Natl Acad Sci U S A 89, 6756-6760.

Boyd, A. \& Simon, M. I. (1980). Multiple electrophoretic forms of methyl-accepting chemotaxis proteins generated by stimuluselicited methylation in Escherichia coli. J Bacteriol 143, 809-815.

Bulter, S. L. \& Falke, J. J. (1998). Cysteine and disulfide scanning reveals two amphiphilic helices in the linker region of the aspartate chemoreceptor. Biochemistry 37, 10746-10756.
Chelsky, D. \& Dahlquist, F. W. (1980). Structural studies of methylaccepting chemotaxis proteins of Escherichia coli: evidence for multiple methylation sites. Proc Natl Acad Sci US A 77, 24342438.

Danielson, M. A., Bass, R. B. \& Falke, J. J. (1997). Cysteine and disulfide scanning reveals a regulatory $\alpha$-helix in the cytoplasmic domain of the aspartate receptor. J Biol Chem 272, 32878-32888.

DeFranco, A. L. \& Koshland, D. E., Jr (1980). Multiple methylation in processing of sensory signals during bacterial chemotaxis. Proc Natl Acad Sci U S A 77, 2429-2433.

Djordjevic, S. \& Stock, A. M. (1998). Chemotaxis receptor recognition by protein methyltransferase CheR. Nature Struct Biol 5, 446-450.

Engström, P. \& Hazelbauer, G. L. (1980). Multiple methylation of methyl-accepting chemotaxis proteins during adaptation of $E$. coli to chemical stimuli. Cell 20, 165-171.

Falke, J. J., Bass, R. B., Butler, S. L., Chervitz, S. A. \& Danielson, M. A. (1997). The two component signaling pathway of bacterial chemotaxis : a molecular view of signal transduction by receptors, kinases, and adaptation enzymes. Annu Rev Cell Dev Biol 13, 457-512.

Feng, X., Baumgartner, J. W. \& Hazelbauer, G. L. (1997). Highand low-abundance chemoreceptors in Escherichia coli: differential activities associated with closely related cytoplasmic domains. J Bacteriol 179, 6714-6720.

Feng, X., Lilly, A. A. \& Hazelbauer, G. L. (1999). Enhanced function conferred on low abundance chemoreceptor Trg by a methyltransferase-docking site. J Bacteriol 181, 3164-3171.

Gegner, J. A., Graham, D. R., Roth, A. F. \& Dahlquist, F. W. (1992). Assembly of an MCP, CheW, and kinase CheA complex in the bacterial chemotaxis signal transduction pathway. Cell 70, 975-982.

Hazelbauer, G. L. \& Engström, P. (1980). Parallel pathways for transduction of chemotactic signals in Escherichia coli. Nature 283, 98-100.

Hess, J. F., Oosawa, K., Kaplan, N. \& Simon, M. I. (1988). Phosphorylation of three proteins in the signaling pathway of bacterial chemotaxis. Cell 53, 79-87.

Iwama, T., Homma, M. \& Kawagishi, I. (1997). Uncoupling of ligand-binding affinity of the bacterial serine chemoreceptor from methylation- and temperature-modulated signaling states. $J$ Biol Chem 272, 13810-13815.

Kim, K. K., Yokota, H. \& Kim, S.-H. (1999). Four-helical-bundle structure of the cytoplasmic domain of a serine chemotaxis receptor. Nature 400, 787-792.

Kondoh, H., Ball, C. B. \& Adler, J. (1979). Identification of a methyl-accepting chemotaxis protein for the ribose and galactose chemoreceptors of Escherichia coli. Proc Natl Acad Sci US A 76, 260-264.

Landt, O., Grunert, H. P. \& Hahn, U. (1990). A general method for rapid site-directed mutagenesis using the polymerase chain reaction. Gene 96, 125-128.

Le Moual, H. \& Koshland, D. E., Jr (1997). Methylation of the Escherichia coli chemotaxis receptors: intra- and interdimer mechanisms. Biochemistry 36, 13441-13448.

Li, J., Li, G. \& Weis, R. M. (1997). The serine receptor from Escherichia coli is methylated through an inter-dimer process. Biochemistry 36, 11851-11857.

Liu, Y., Levit, M., Lurz, R., Surette, M. G. \& Stock, J. B. (1997). Receptor-mediated protein kinase activation and the mechanism of transmembrane signaling in bacterial chemotaxis. EMBO J 16, 7231-7240. 
Lupas, A. \& Stock, J. (1989). Phosphorylation of an N-terminal regulatory domain activates the $\mathrm{CheB}$ methylesterase in bacterial chemotaxis. J Biol Chem 264, 17337-17342.

Lybarger, S. R. \& Maddock, J. R. (2000). Differences in the polar clustering of the high- and low-abundance chemoreceptors of Escherichia coli. Proc Natl Acad Sci U S A 97, 8057-8062.

Maddock, J. R. \& Shapiro, L. (1993). Polar location of the chemoreceptor complex in the Escherichia coli cell. Science 259, 1717-1723.

Manson, M. D. (1992). Bacterial motility and chemotaxis. $A d v$ Microb Physiol 33, 277-346.

Milligan, D. L. \& Koshland, D. E., Jr (1988). Site-directed cross linking: establishing the dimeric structure of the aspartate receptor of bacterial chemotaxis. J Biol Chem 263, 6268-6275.

Nishiyama, S., Nara, T., Imae, Y., Homma, M. \& Kawagishi, I. (1997). Thermosensing properties of mutant aspartate receptors having methyl-accepting sites substituted multiply or singly with alanine. J Bacteriol 179, 6573-6580.

Nishiyama, S., Umemura, T., Nara, T., Homma, M. \& Kawagishi, I. (1999). Conversion of a bacterial warm sensor to a cold sensor by methylation of a single residue in the presence of an attractant. Mol Microbiol 32, 357-365.

Okumura, H., Nishiyama, S., Sasaki, A., Homma, M. \& Kawagishi, I. (1998). Chemotactic adaptation is altered by changes in the Cterminal sequence conserved among the major methyl accepting chemoreceptors. J Bacteriol 180, 1862-1868.

Oosawa, K. \& Simon, M. I. (1986). Analysis of mutations in the transmembrane region of aspartate chemoreceptor in Escherichia coli. Proc Natl Acad Sci U S A 83, 6930-6934.

Parkinson, J. S. (1993). Signal transduction schemes of bacteria. Cell 73, 857-871.

Schuster, S. C., Swanson, R. V., Alex, L. A., Bourret, R. B. \& Simon, M. I. (1993). Assembly and function of a quaternary signal transduction complex monitored by surface plasmon resonance. Nature 365, 343-347.

Shiomi, D., Okumura, H., Homma, M. \& Kawagishi, I. (2000). The aspartate chemoreceptor Tar is effectively methylated by binding to the methyltransferase mainly through hydrophobic interaction. Mol Microbiol 36, 132-140.

Shiomi, D., Zhulin, I. B., Homma, M. \& Kawagishi, I. (2002). Dual recognition of the bacterial chemoreceptor by chemotaxis-specific domains of the CheR methyltransferase. J Biol Chem (in press).

Simms, S. A., Stock, A. M. \& Stock, J. B. (1987). Purification and characterization of $S$-adenosylmethionine:glutamyl methyltransferase that modifies membrane chemoreceptor proteins in bacteria. J Biol Chem 262, 8537-8543.

Springer, M. S., Goy, M. F. \& Adler, J. (1977). Sensory transduction in Escherichia coli: two complementary pathways of information processing that involve methylated proteins. Proc Natl Acad Sci U S A 74, 3312-3316.
Springer, W. R. \& Koshland, D. E., Jr (1977). Identification of a protein methyltransferase as the che R gene product in the bacterial sensing system. Proc Natl Acad Sci U S A 74, 533-537.

Stock, A. M., Wylie, D. C., Mottonen, J. M., Lupas, A. M., Ninfa, E. G., Ninfa, A. J., Schutt, C. E. \& Stock, J. B. (1988). Phosphoproteins involved in bacterial signal transduction. Cold Spring Harbor Symp Quant Biol 53, 49-57.

Stock, J. B. \& Surette, M. G. (1996). Chemotaxis. In Escherichia coli and Salmonella typhimurium: Cellular and Molecular Biology, 2nd edn, pp. 1103-1129. Edited by F. C. Neidhardt \& others. Washington, DC: American Society for Microbiology.

Tatsuno, I., Homma, M., Oosawa, K. \& Kawagishi, I. (1996). Signaling by the Escherichia coli aspartate chemoreceptor Tar with a single cytoplasmic domain per dimer. Science 274, 423-425.

Trammell, M. A. \& Falke, J. J. (1999). Identification of a site critical for kinase regulation on the central processing unit (CPU) helix of the aspartate receptor. Biochemistry 38, 329-336.

Umemura, T., Tatsuno, I., Shibasaki, M., Homma, M. \& Kawagishi, I. (1998). Intersubunit interaction between transmembrane helices of the bacterial aspartate chemoreceptor homodimer. J Biol Chem 273, 30110-30115.

Wang, E. A. \& Koshland, D. E., Jr (1980). Receptor structure in the bacterial sensing system. Proc Natl Acad Sci U S A 77, 7157-7161.

Weerasuriya, S., Schneider, B. M. \& Manson, M. D. (1998). Chimeric chemoreceptors in Escherichia coli: signaling and methylation properties of Tar-Tap and Tap-Tar hybrids. J Bacteriol 180, 914-920.

Welch, M., Oosawa, K., Aizawa, S.-I. \& Eisenbach, M. (1993). Phosphorylation-dependent binding of a signal molecule to the flagellar switch of bacteria. Proc Natl Acad Sci USA 90, 8787-8791.

Wolfe, A. J. \& Berg, H. C. (1989). Migration of bacteria in semisolid agar. Proc Natl Acad Sci U S A 86, 6973-6977.

Wolfe, A. J., Conley, M. P., Kramer, T. J. \& Berg, H. C. (1987). Reconstitution of signaling in bacterial chemotaxis. J Bacteriol 169, 1878-1885.

Wu, J., Li, J., Li, G., Long, D. G. \& Weis, R. M. (1996). The receptor binding site for the methyltransferase of bacterial chemotaxis is distinct from the sites of methylation. Biochemistry 35, 49844993.

Wylie, D., Stock, A. M., Wong, C.-Y. \& Stock, J. (1988). Sensory transduction in bacterial chemotaxis involves phosphotransfer between Che proteins. Biochem Biophys Res Commun 151, 891-896.

Yamamoto, K., Macnab, R. M. \& Imae, Y. (1990). Repellent response functions of the $\operatorname{Trg}$ and Tap chemoreceptors of Escherichia coli. J Bacteriol 172, 383-388.

Received 28 February 2002; revised 4 June 2002; accepted 21 June 2002. 